UDC 621.3.026.5

SCOPUS CODE 2101

https://doi.org/10.36073/1512-0996-2020-4-105-112

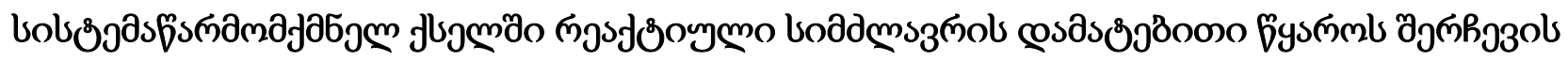

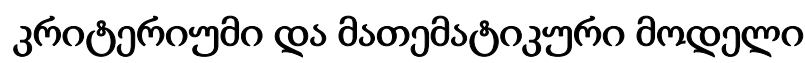

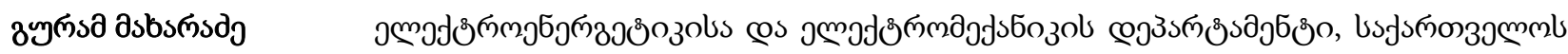

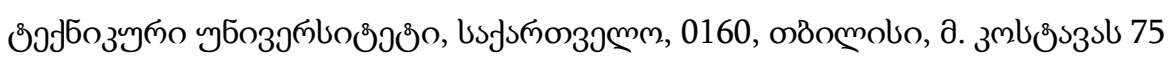

E-mail: guram.makharadze@gse.com.ge

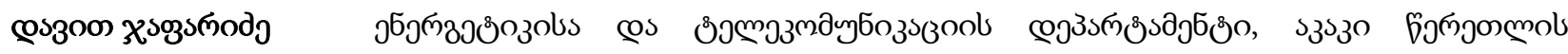

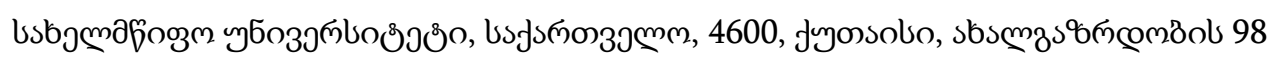
E-mail: datojaparidze1995@gmail.com

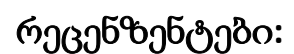

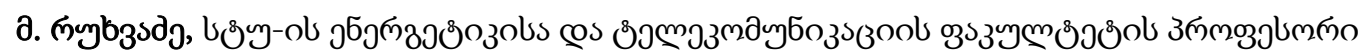

E-mail: misharuch@gmail.com

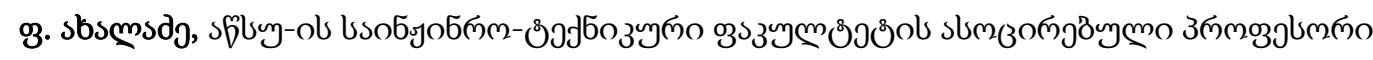

E-mail: pridon.akhaladze@atsu.edu.ge

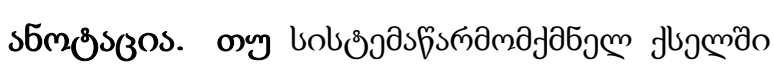

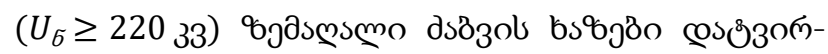

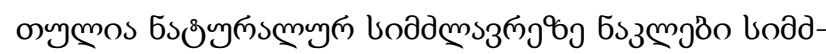

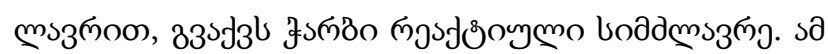

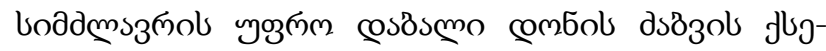

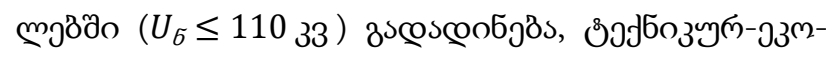

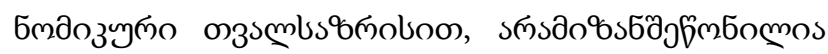

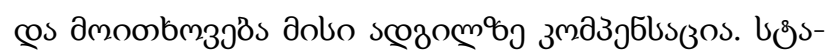

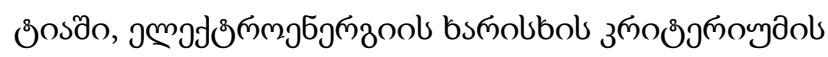

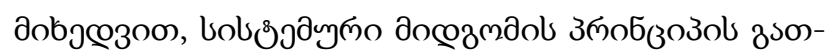

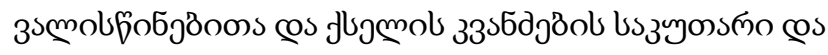

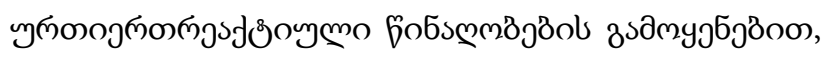

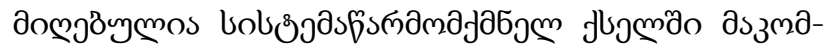

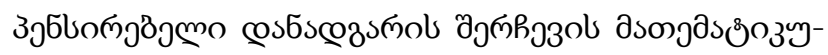
๓о дмеумо.

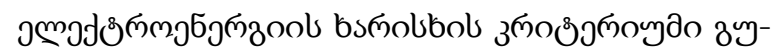

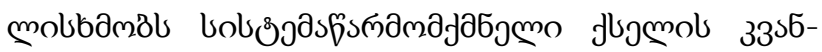

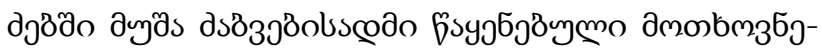

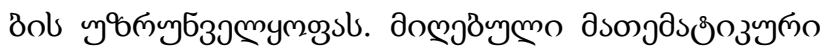

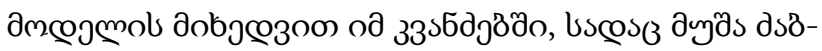

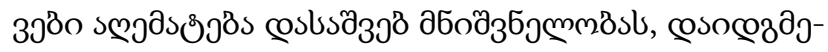

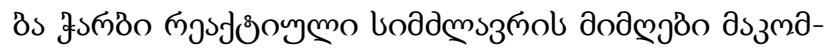

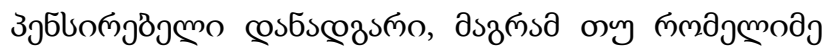

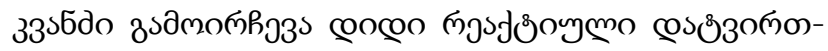

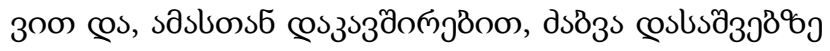




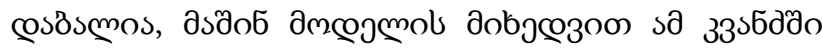

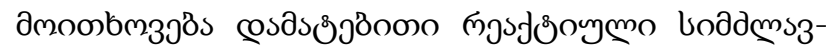

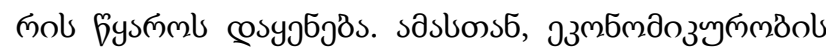

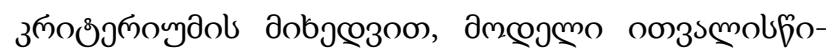

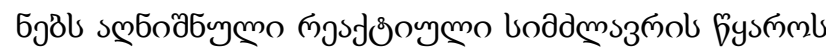

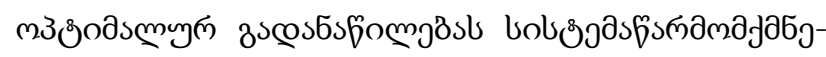

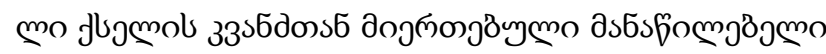

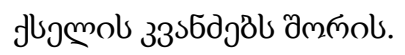

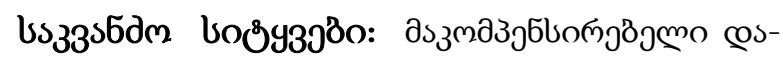

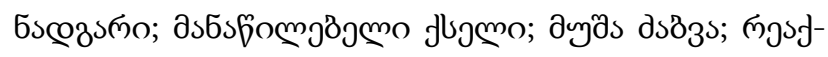

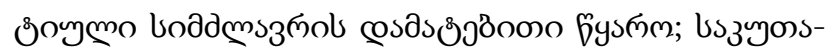

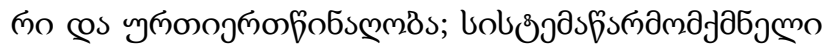
孔๖лмо.

\section{gjlsзzsmo}

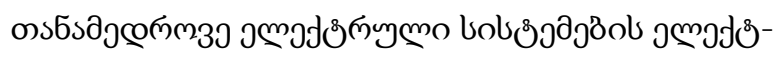

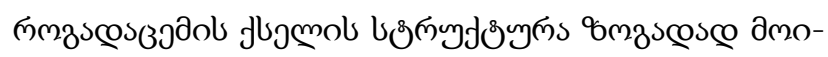

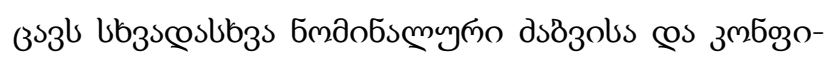

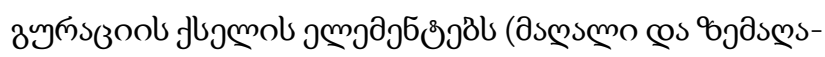

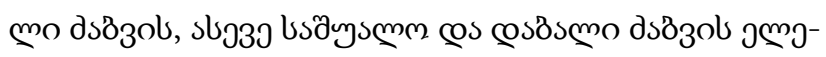

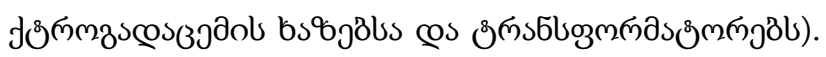

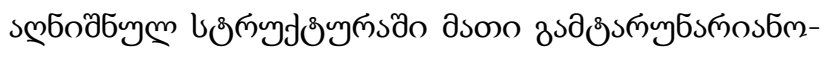

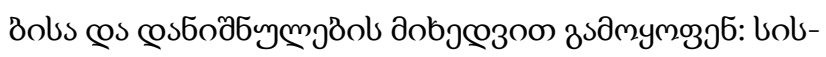

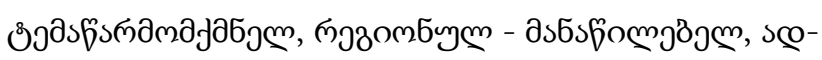

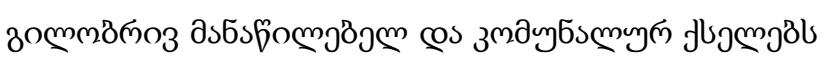
(byñ. 1) [1].

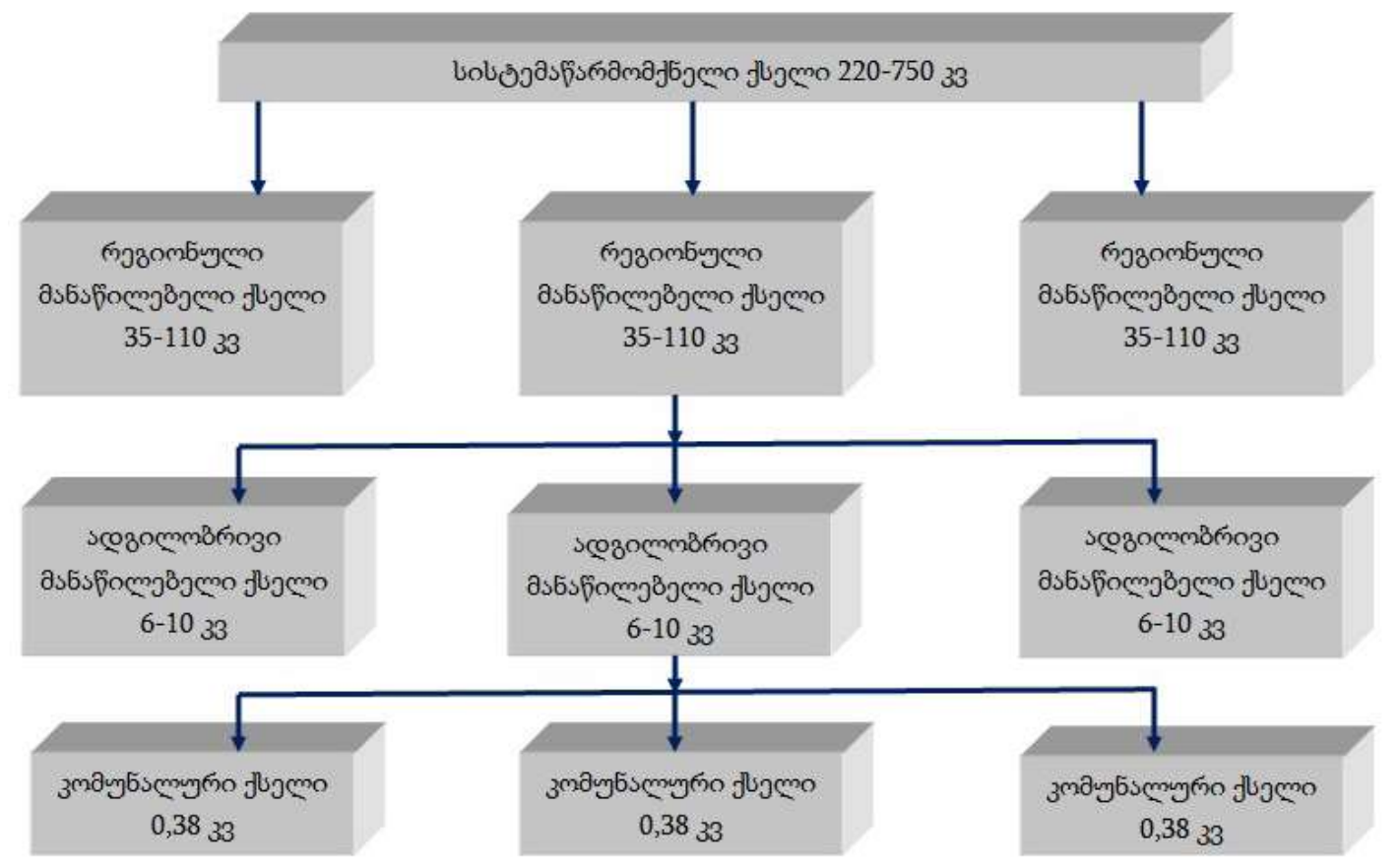

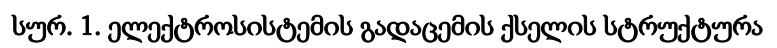

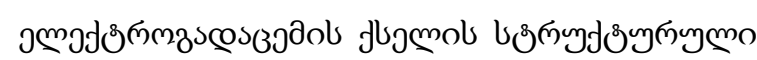

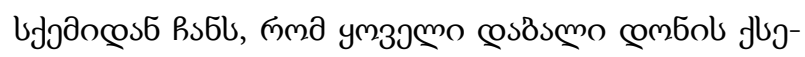

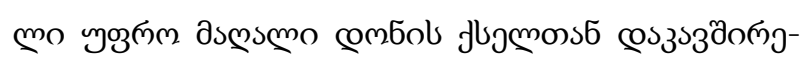

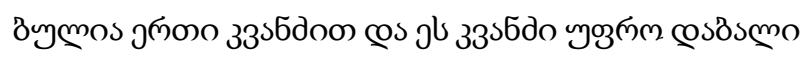




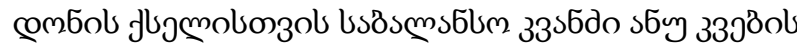

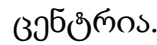

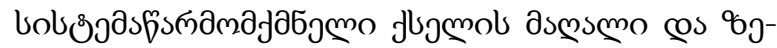

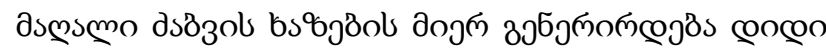

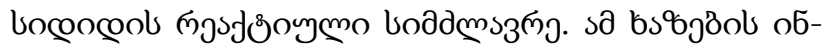

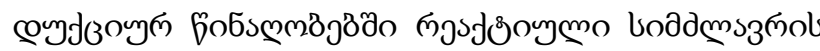

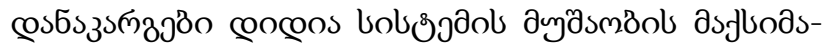

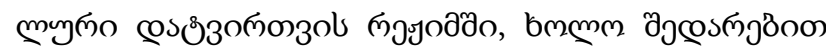

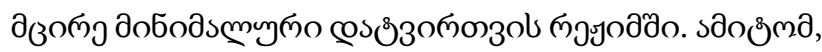

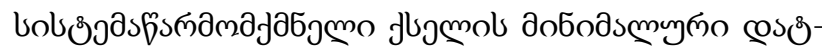

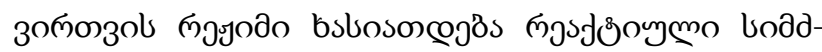

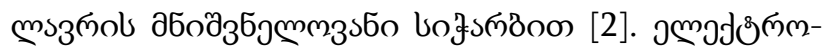

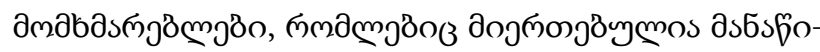

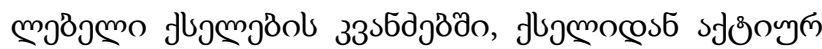

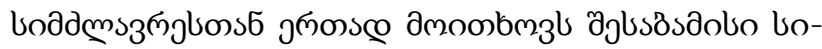

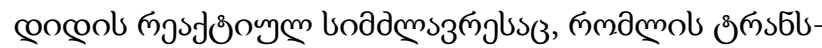

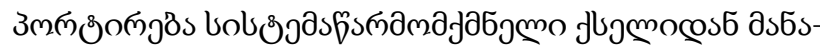

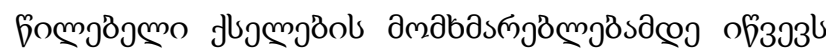

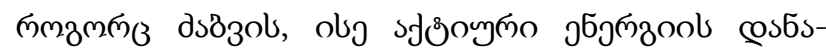

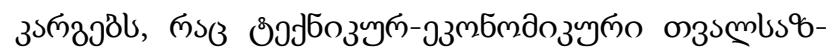

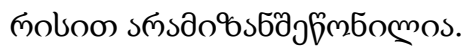

\section{domonscen 5sformo}

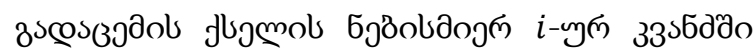

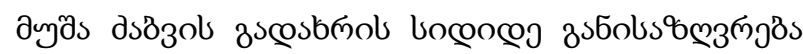
зsambsbygmgo̊on [3]:

$$
\Delta U_{i}=\frac{1}{U_{6}} \cdot \sum_{j=1}^{n}\left(P_{j} R_{i j}+Q_{j} X_{i j}\right) .
$$

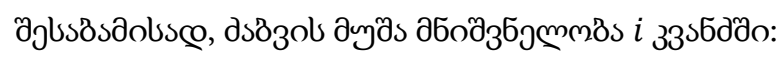

$$
U_{i}=U_{0}+\frac{1}{U_{6}} \cdot \sum_{j=1}^{n}\left(P_{j} R_{i j}+Q_{j} X_{i j}\right),
$$

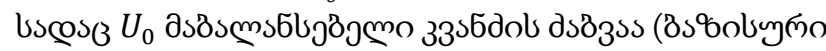

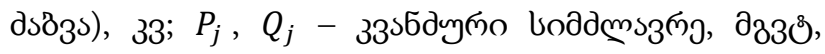

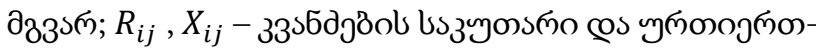

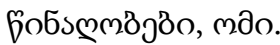

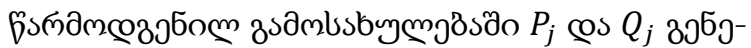

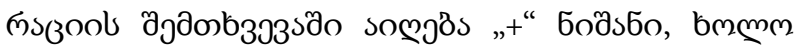

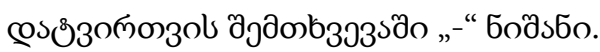

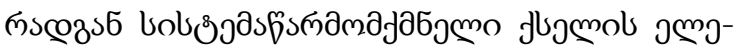

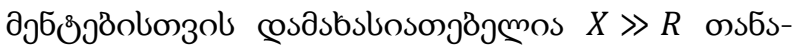

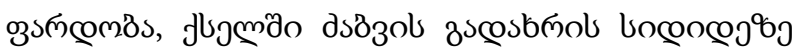

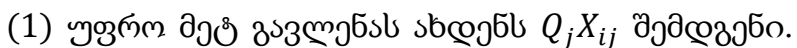

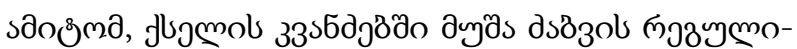

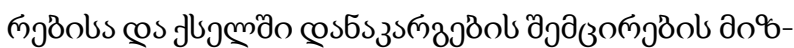

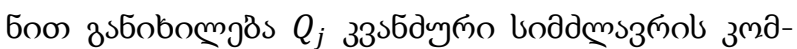

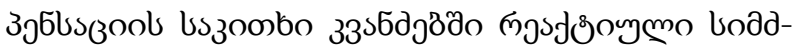

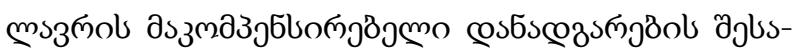

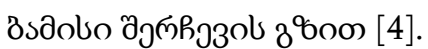

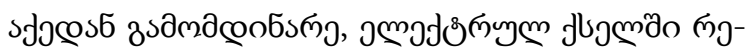

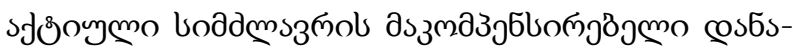

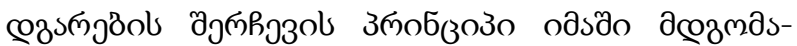

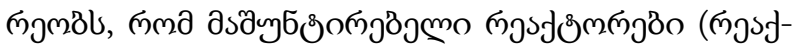

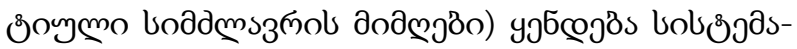

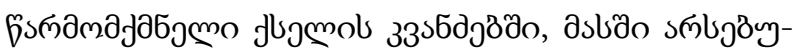

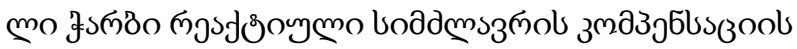

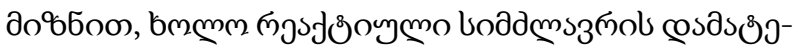

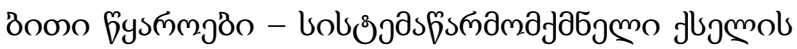

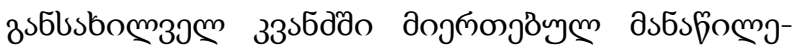

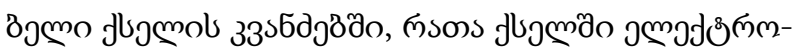

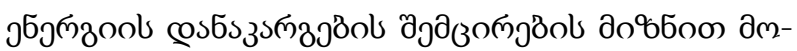

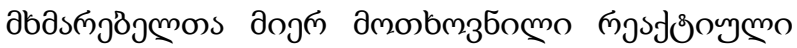

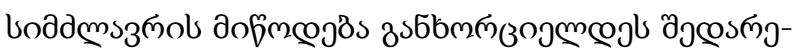

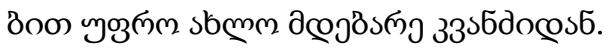

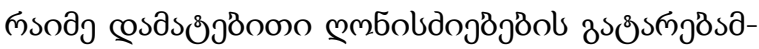

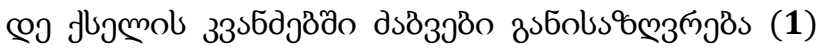

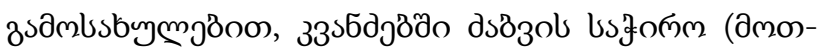




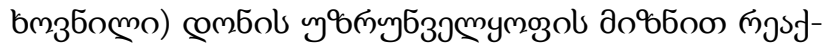

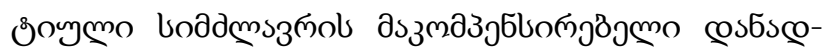

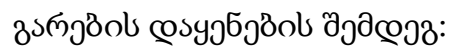

$$
U_{0}+\frac{1}{U_{6}} \cdot \sum_{j=1}^{n}\left(P_{j} R_{i j}+\left(Q_{j}+Q_{3 j}\right) X_{i j}\right)=U_{i, \text { वсosb }}
$$

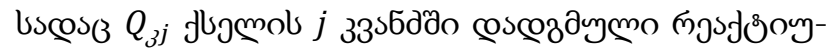

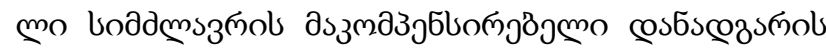

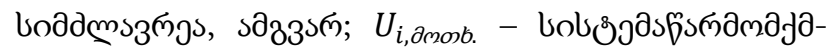

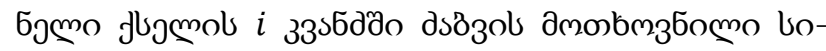
๓ому.

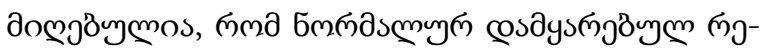

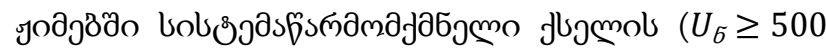

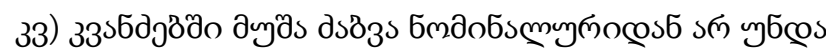

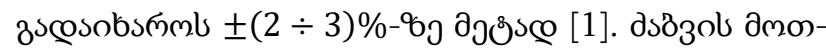

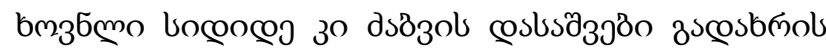

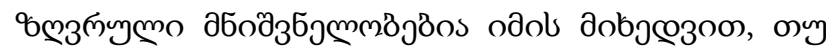

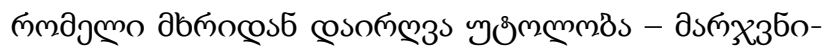

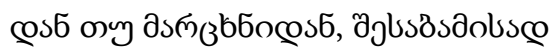

$$
(0,97 \div 0,98) U_{6}=U_{i, \text { дмоь }}
$$

Qs

$$
(1,02 \div 1,03) U_{6}=U_{i, \text { dmob }}
$$

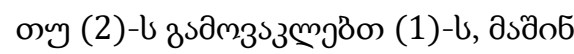

$$
\sum_{j=1}^{n} Q_{3 j} X_{i j}=U_{6} \cdot\left(U_{i, \text { amoob. }}-U_{i}\right) .
$$

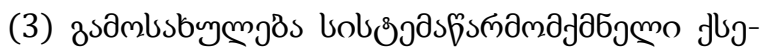

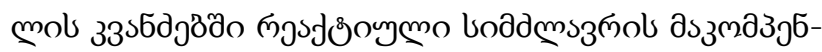

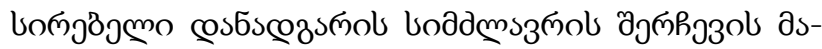

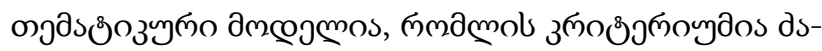

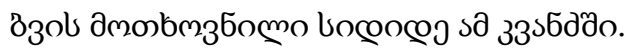

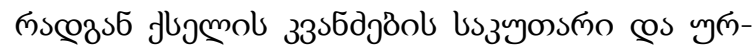

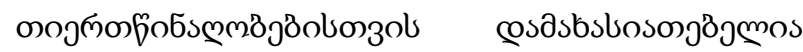

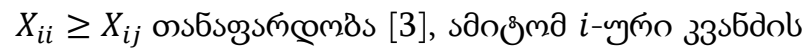

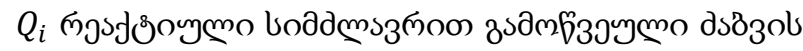

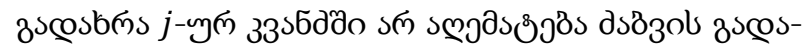

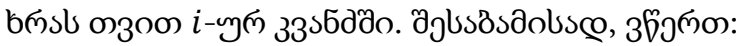

$$
\frac{Q_{i} X_{i j}}{U_{6}} \leq \frac{Q_{i} X_{i i}}{U_{6}}
$$

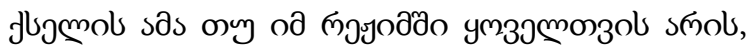

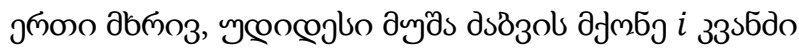

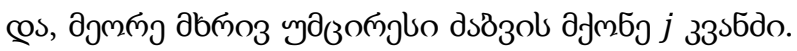
оу jl ds

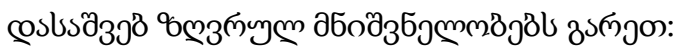

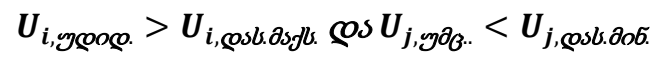

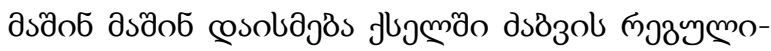

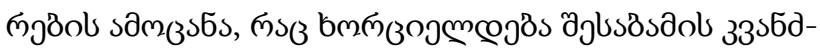

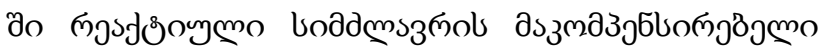

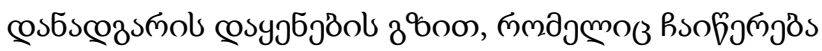

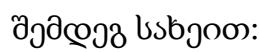

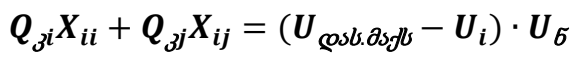

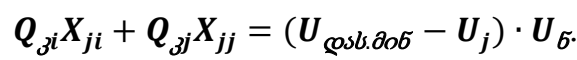

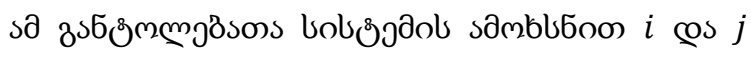

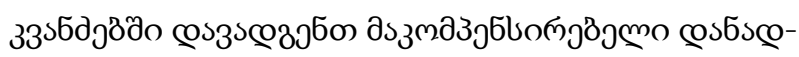

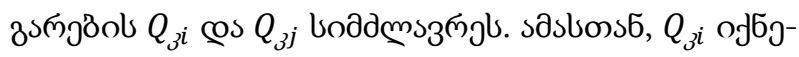

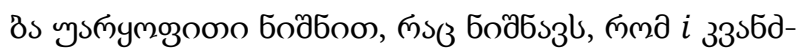

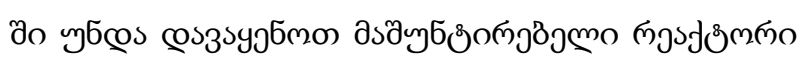

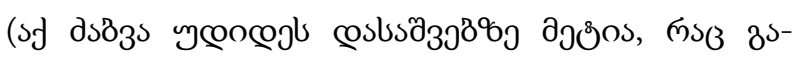

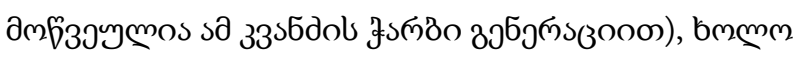

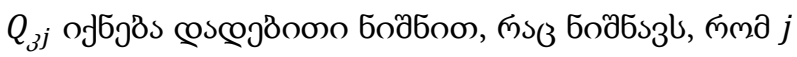

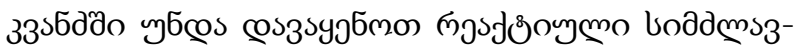

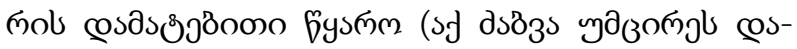

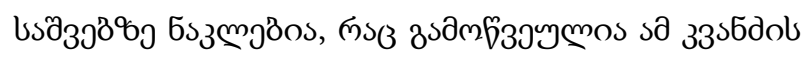

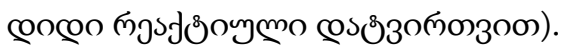

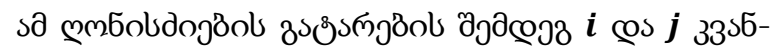

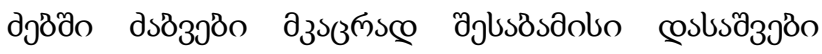

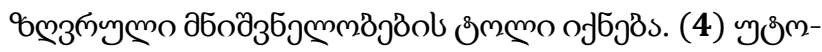

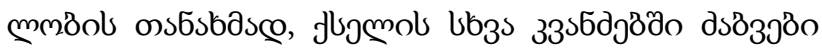

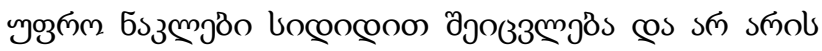




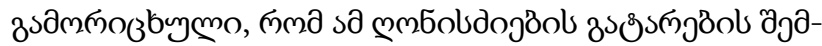

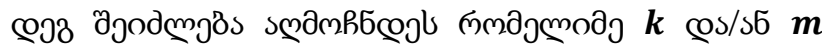

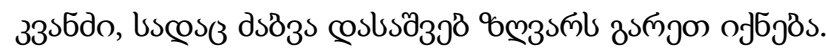

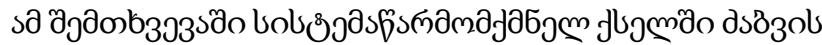

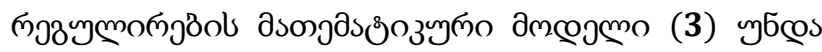

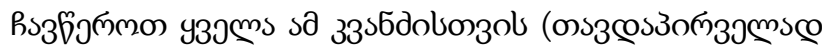

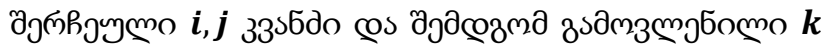
cos/s6 $\boldsymbol{m}_{33}$ s6do):

$$
\begin{aligned}
& Q_{3 i} X_{i i}+Q_{3 j} X_{i j}+Q_{3 k} X_{i k}+Q_{3 m} X_{i m}= \\
& =\left(U_{\text {cost. } \partial s f l s}-U_{i}\right) \cdot U_{\sigma} \text {; } \\
& Q_{3 i} X_{j i}+Q_{3 j} X_{j j}+Q_{3^{k}} X_{j k}+Q_{3 m} X_{j m}= \\
& =\left(U_{\text {cost.доб }}-U_{j}\right) \cdot U_{6} \text {; } \\
& Q_{3 i} X_{k i}+Q_{3 j} X_{k j}+Q_{3 k} X_{k k}+Q_{3 m} X_{k m}= \\
& =\left(U_{\text {cosl.asfl }}-U_{k}\right) \cdot U_{6} \text {; } \\
& Q_{3 i} X_{m i}+Q_{3 j} X_{m j}+Q_{3 k} X_{m k}+Q_{3 m} X_{m m}= \\
& =\left(U_{\text {œosk. } \partial \circ \sigma}-U_{m}\right) \cdot U_{6 \text {. }}
\end{aligned}
$$

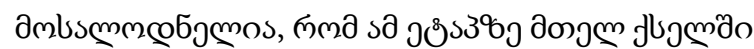

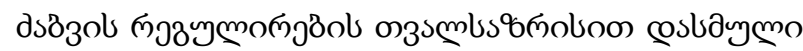

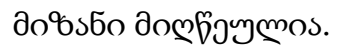

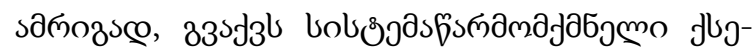

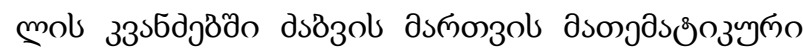

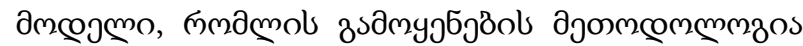
วృдюวзо:

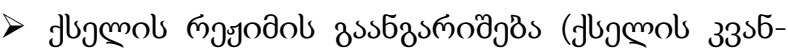

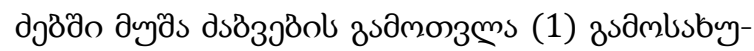

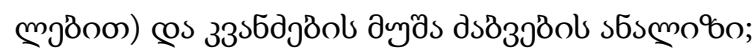

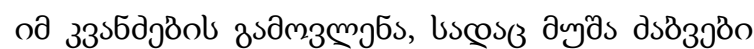

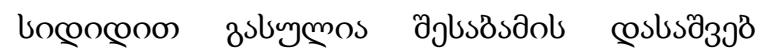

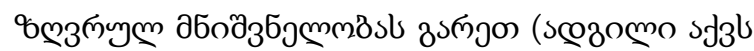
(5) эช্mmmösl);

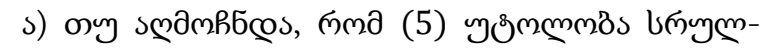

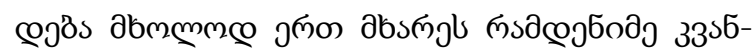

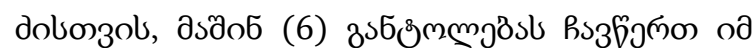

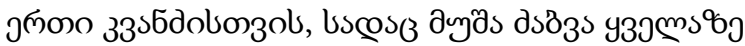

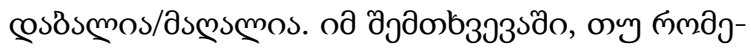

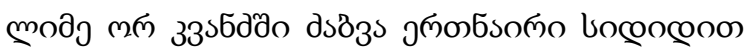

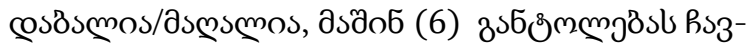

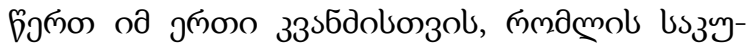

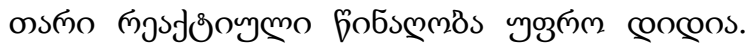

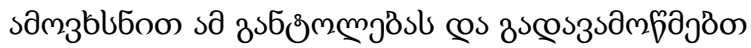

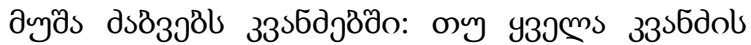

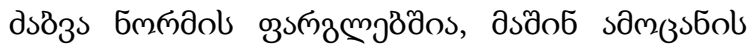

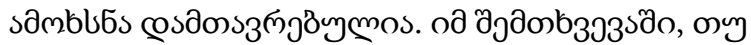

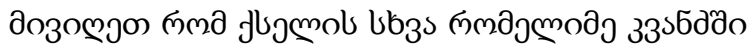

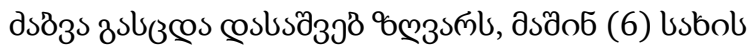

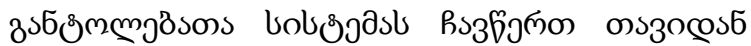

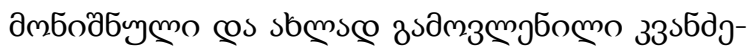

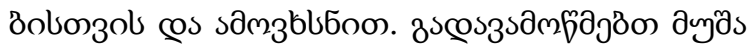

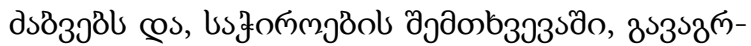

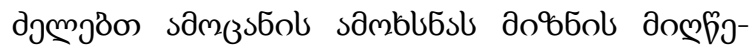
Зรวณ๐.

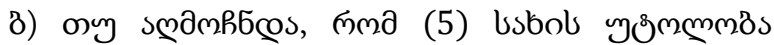

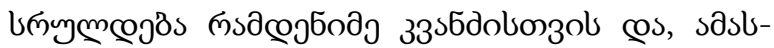

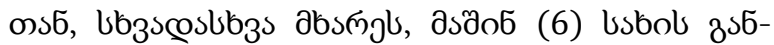

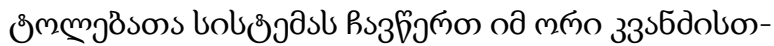

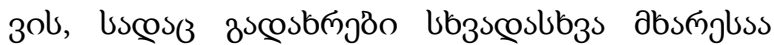

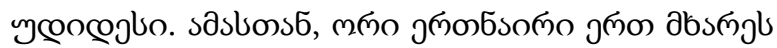

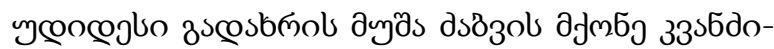

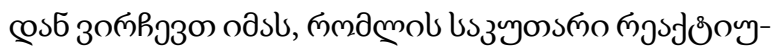

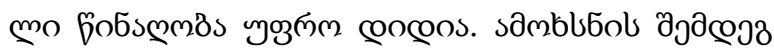

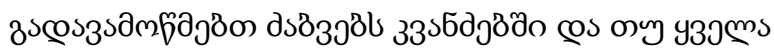

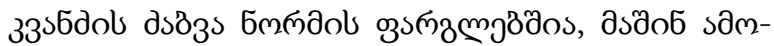

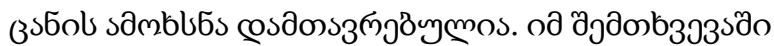

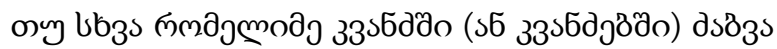

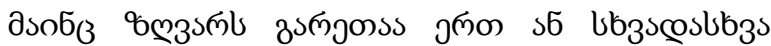

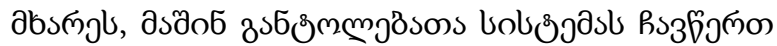




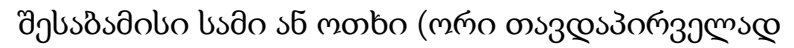

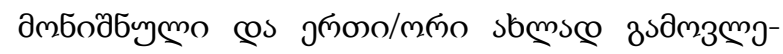

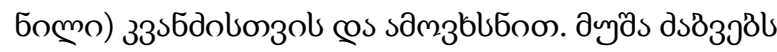

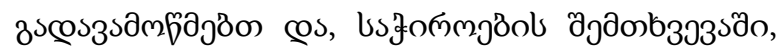

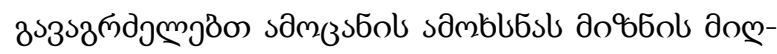
бозงамอง.

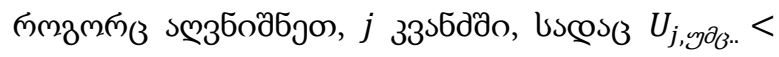

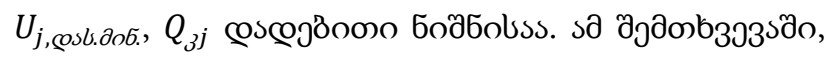

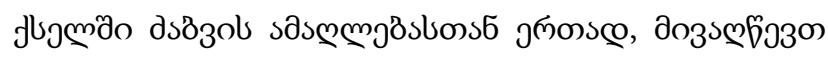

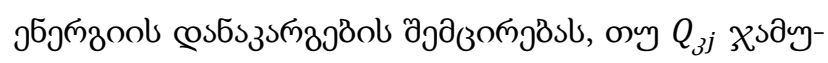

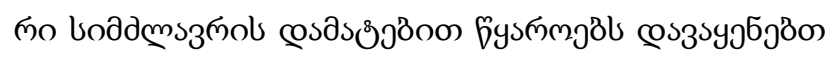

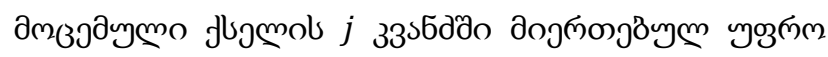

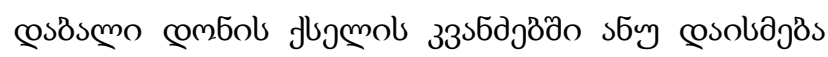

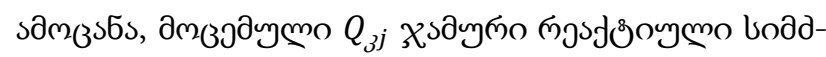

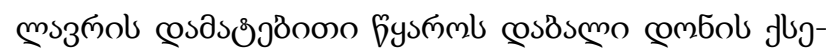

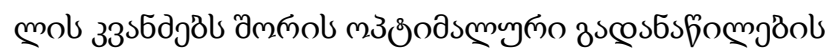

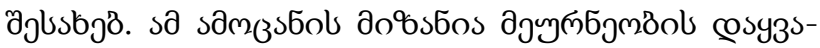

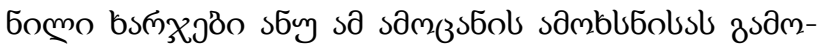

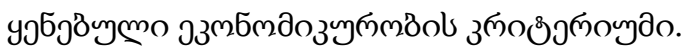

\section{cosls $336 s$}

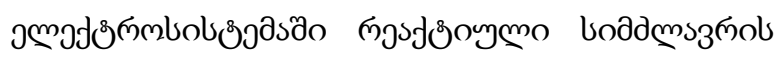

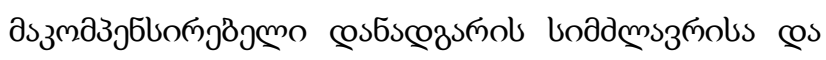

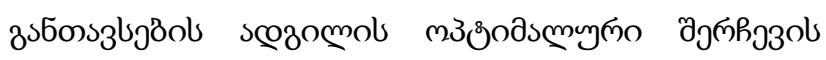

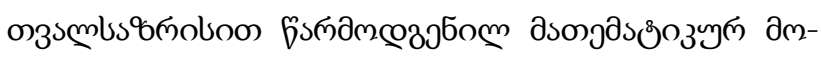

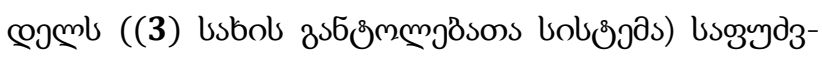

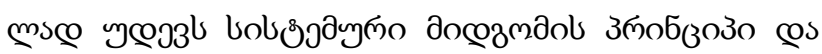

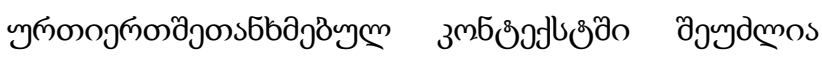

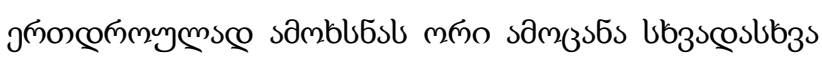

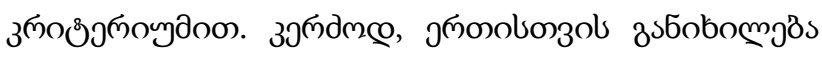

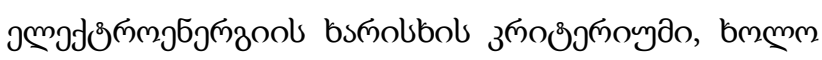

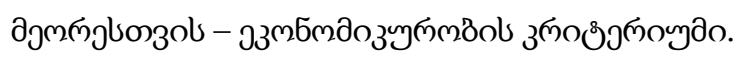

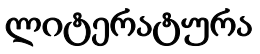

1. Venikov V.A., Glazunov A.A., Zhukov L.S., Soldatkina L.A. Electrical systems.Vol.2. Electric networks. Edited by V.A. Venikov. 1971. (In Russian).

2. Glazunov A.A. Electric network and systems. M.: "Gosenergoizdat”. 1960. (In Russian).

3. Makharadze G. Management and optimization of energy regimes. Publishing house „Technical-University“. Tbilisi. 2005. (In Georgian).

4. Makharadze G. Electroenergetics (electrical) systems. Publishing house „Universal“. Tbilisi. 2015. (In Georgian). 
UDC 621.3.026.5

SCOPUS CODE 2101

https://doi.org/10.36073/1512-0996-2020-4-105-112

\title{
Selection criteria and a mathematical model for the additional source of reactive power in a backbone network
}

\author{
Guram Makharadze Department of Electrical Power Engineering and Electromechanics, Georgian \\ Technical University, 75 M. Kostava str., 0160 Tbilisi, Georgia \\ E-mail: guram.makharadze@gse.com.ge \\ David Japaridze Department of Energy and Telecommunications, Akaki Tsereteli State University, 98 \\ Youth str., 4600 Kutaisi, Georgia \\ E-mail: atojaparidze1995@gmail.com
}

\section{Reviewers:}

M. Rukhvadze, Professor, Faculty of Power Engineering and Telecommunication, GTU

E-mail: misharuch@gmail.com

P. Akhaladze, Associate Professor, Faculty of Technical Engineering, ATSU

E-mail: pridon.akhaladze@atsu.edu.ge

Abstract. In a backbone network $\left(U_{n} \geq 220 \mathrm{kv}\right)$, when the high-voltage lines are loaded with power less than natural power, we have excess reactive power. Supplying this power into the lower-voltage networks $\left(U_{n} \leq 110 \mathrm{kv}\right)$ would be technically and economically unfeasible and requires compensation on site. In the article, in accordance with the electricity quality criterion, and taking into account the principle of a systemic approach, and using the self- and mutually reactive impedances of the network junction points, a mathematical model for selecting a compensating device in a backbone network is adopted.

The quality criterion of electricity involves enforcement of requirements for the operating voltages in the junction points of a backbone network. According to the obtained mathematical model, in the junction points nodes where the operating voltages exceed their permitted values, there will be installed the compensating devices for receiving excess reactive power. However if any junction point has a high reactive load and the voltage, in this context, is below its permitted value, then, according to a model, there is a need for installing the source of reactive power in this junction point. Herewith, according to economic criterion, the model envisages the optimal redistribution of mentioned source of reactive power between the junction points of a distribution network connected to backbone network junction point.

Key words: Additional reactive power source; backbone network; compensating device; distribution network; operating voltage; self- and mutually reactive impedances.

ISSN 1512-0996

www.shromebi.gtu.ge 
UDC 621.3.026.5

SCOPUS CODE 2101

https://doi.org/10.36073/1512-0996-2020-4-105-112

\title{
Критерии выбора дополнительного источника реактивной мощности в системообразующей сети и математическая модель
}

Гурам Махарадзе

Давид Джапаридзе департамент электроэнергетики и электромеханики, Грузинский технический университет, Грузия, 0160, Тбилиси, ул. М. Костава, 75

E-mail: guram.makharadze@gse.com.ge департамент энергетики и телекоммуникации, Государственный университет им. Акакия Церетели, Грузия, 4600, Кутаиси, ул. Молодежи, 98

E-mail: atojaparidze1995@gmail.com

\section{Рецензенты:}

М. Рухвадзе, профессор факультета энергетики и телекоммуникации ГТУ

E-mail: misharuch@gmail.com

Ф. Ахаладзе, ассоциированный профессор инженерно-технического факультета ГУАЦ

E-mail: pridon.akhaladze@atsu.edu.ge

Аннотация. В системообазующей сети ( $U_{\mathrm{H}} \geq 220$ кв), при загрузке линий сверхвысокого напряжения меньшей натуральной, имеется избыток реактивной мощности. Переток этой мощности в сети низкого напряжения $\left(U_{\mathrm{H}} \leq 110\right.$ кв), технико-экономически нецелесообразно и требуется ее компенсация. В статье по критерию качества электроэнергии, с учетом принципа системного подхода и с применением собственных и взаимно реактивных сопротивлений узлов, получена математическая модель выбора компенсирующих устройств в системообразующей сети.

Указанный в статье критерий качества электроэнергии подразумевает обеспечению узловых напряжений в пределах допустимого. Согласно полученной математической модели, в узлах системообразующей сети, где рабочие напряжения превышают допустимое значение, будут установлены приемники избыточной реактивной мощности. Однако, в узле, где имеется большая реактивная нагрузка и, в связи с этим, рабочее напряжение ниже допустимого, требуется установка дополнительного источника реактивной мощности (ИРМ). При этом, по критерию экономичности, модель предусматривает оптимальное перераспределение источника реактивной мощности между узлами региональной сети, подключенной к узлу системо-образующей сети.

Ключевые слова: дополнительный источник реактивной мощности (ИРМ); компенсирующие устройства; рабочее напряжение; распределительная сеть; системообразующая сеть; собственное и взаимное сопротивление.

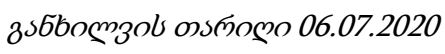 \\ ğдmbз

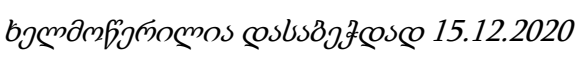

